\title{
Single-mode direct-ultraviolet-written channel waveguide laser in neodymium-doped silica on silicon
}

\author{
D. A. Guilhot, G. D. Emmerson, C. B. E. Gawith, S. P. Watts, D. P. Shepherd, R. B. Williams, and P. G. R. Smith \\ Optoelectronics Research Centre, University of Southampton, Southampton SO17 1BJ, UK
}

Received October 23, 2003

\begin{abstract}
A waveguide laser with a neodymium-doped silica core is fabricated on a silicon substrate by a combination of flame hydrolysis deposition, solution doping, and direct UV writing. The neodymium-ion concentration is estimated to be $\sim 8000$ parts in $10^{6}$. The propagation loss around $1.05 \mu \mathrm{m}$ is $<0.8 \mathrm{~dB} / \mathrm{cm}$. Lasing in the range $1048-1056 \mathrm{~nm}$ and $1356 \pm 1 \mathrm{~nm}$ is observed. A slope efficiency of $33 \%$ for a high-reflectivity output coupler and a threshold of $4 \mathrm{~mW}$ of absorbed power for a $25 \%$ output coupler are measured for the ${ }^{4} F_{3 / 2} \rightarrow{ }^{4} I_{11 / 2}$ transition. (C) 2004 Optical Society of America

OCIS codes: $140.3530,230.7390,160.6030$.
\end{abstract}

Rare-earth-doped glass waveguide lasers have been the subject of a considerable amount of investigation recently, and a number of fabrication techniques have been used to realize this kind of device. ${ }^{1-3}$ One of the early successes ${ }^{4}$ was achieved with a combination of flame hydrolysis deposition (FHD), solution doping, and reactive ion etching. Silica on silicon has the important advantage of allowing simple mode matching to standard telecommunication fiber. ${ }^{5}$

On the basis of a localized change in the refractive index induced by short-wavelength radiation, direct UV writing allows waveguide structures to be drawn into a photosensitive core layer by computer-controlled scanning of the layer under a focused UV beam, ${ }^{6}$ eliminating the development time and costs associated with repeated photolithographic steps. In previous work, direct UV writing has been proved to provide low-loss channel waveguides in germanium-doped silica on silicon while allowing rapid prototyping toward the fabrication of low-cost integrated optical components. In addition, either hydrogen or deuterium loading has long been used to enhance the photosensitivity of germanosilicates, ${ }^{6}$ increasing the photosensitive response from any given dopant level.

The inclusion of phosphorus within the composition increases the doping level of rare-earth elements that can be incorporated in the layer without devitrification. ${ }^{7}$ By tailoring the amount of germanium, phosphorus, and neodymium, we managed to produce a laser in a photosensitive core layer. The substrate was fabricated with the FHD and solution-doping techniques. Channel waveguides were formed in the neodymium-doped photosensitive core layer with the direct-UV-writing technique. In this Letter we present the first demonstration, to our knowledge, of a direct-UV-written waveguide laser in neodymiumdoped silica on silicon. The aim was to create a planar waveguide laser in the silica-on-silicon format, which has good lasing characteristics but is also suitably photosensitive to allow UV definition of waveguides.

The samples used for this experiment were threelayer silica-on-silicon substrates deposited by FHD. The underclad silica was doped with germanium, phosphorus, and boron, while a low-density oxide soot produced from chlorides of silicon, germanium, phosphorus, and boron was deposited on this underclad layer to form the core layer. The amount of germanium in the core was measured to be $4.1 \mathrm{wt} . \%$ and the amount of phosphorus was 12.3 wt. \% by secondary ion mass spectroscopy measurements. Each sample was placed in a furnace at a temperature of $875^{\circ} \mathrm{C}$ for a duration of $15 \mathrm{~min}$ to presinter the soot. The neodymium was introduced inside the core layer by solution doping ${ }^{4}$ with $0.011 \mathrm{M}$ of $\mathrm{NdCl}$ in methanol. After drying, the soot layer was sintered in the furnace at $1225^{\circ} \mathrm{C}$ for an hour to produce a fully dense glass layer. It should be noted that doping the soot with neodymium can produce various effects in the core glass, from devitrification to nonconsolidation of the layer. Modifying the presintering and consolidation parameters allowed the amount of phosphorus, germanium, and neodymium in the layer to be tailored to produce an efficient composition. An overclad layer was then deposited by FHD as before. The amount of boron and phosphorus in each layer was modified to match the refractive index of the layers and to decrease the consolidation temperature for each subsequent layer.

Hydrogen loading of the sample at 150 bars for $>5$ days was performed to increase the sample photosensitivity. Direct UV writing into the photosensitive core layer was performed with a frequency-doubled argon laser at $244 \mathrm{~nm}$. The UV beam was focused to a $3.3-\mu \mathrm{m}$ spot within the core layer of the sample. The sample was translated under the focused UV beam by use of high-precision $x, y$, and $z$ stages, creating a localized positive refractive-index change in the photosensitive material and thus inducing channel confinement (Fig. 1). Optimization of the channel waveguide geometry was performed by adjusting the translation speed of the sample between 5 and $40 \mathrm{~mm} / \mathrm{min}$. It was determined that writing conditions of $5 \mathrm{~mm} / \mathrm{min}$ at $80 \mathrm{~mW}$ were suitable to produce single-mode channel waveguide confinement at $1056 \mathrm{~nm}$.

After UV writing, the sample was cut and both end faces were polished to provide 4.5-, 10-, and 


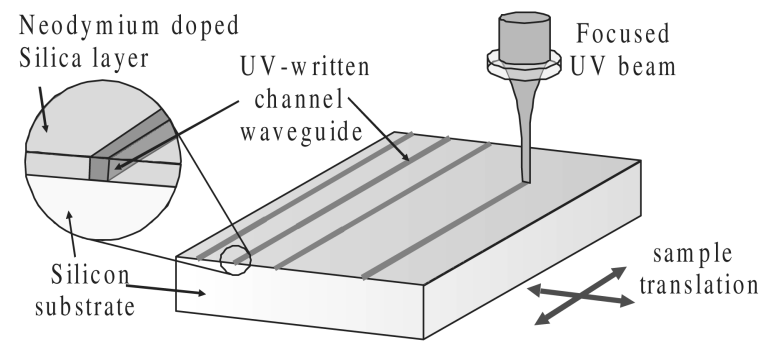

Fig. 1. Schematic diagram of the setup used to directly UV write waveguides into neodymium-doped silica.

18-mm-long samples. Characterization of the laser performance and propagation loss of the device were performed with a tunable (700-850-nm) Ti:sapphire laser for these three sample lengths. Pump radiation was focused into each buried channel waveguide by means of a $10 \times$ microscope objective. The output signal was collected with another $10 \times$ microscope objective and measured by a silicon detector placed after a filter to block transmitted pump light.

The lifetime of the neodymium ${ }^{4} F_{3 / 2} \rightarrow{ }^{4} I_{11 / 2}$ transition was found to be $220 \mu \mathrm{s}$ from fluorescence decay measurements taken with a silicon detector and an oscilloscope. This is similar to the value of $250 \mu \mathrm{s}$ measured by Hattori et al. ${ }^{8}$ for a nonuniform distribution of $\mathrm{Nd}^{3+}$ in planar silica. An optical absorption spectrum of the channel was measured from 450 to $1000 \mathrm{~nm}$ with an optical spectrum analyzer. Absorption peaks were observed at $525,590,680,750,810$, and $890 \mathrm{~nm}$, corresponding to the known spectrum of neodymium. ${ }^{9}$ With the length of the waveguide known, the absorption coefficient in decibels per kilometer per parts per million for different peaks and the height of the absorption peaks in decibels, a value for the neodymium concentration was estimated as 8000 parts per million. Optimization of the launch was performed in relation to the fluorescence measured by a silicon detector. The absorption and launch efficiency were calculated from measurements of the input and output power from the waveguide, with the Ti:sapphire pump laser turned on and off the 810-nm absorption, yielding a launch efficiency of $84 \%$ and absorption values of $50 \%$ for the $4.5-\mathrm{mm}$-long waveguide, $90 \%$ for the 10 -mm-long waveguide, and $100 \%$ for the $18-\mathrm{mm}$-long waveguide. These results suggest an absorption coefficient of approximately $2 \mathrm{~cm}^{-1}$.

Laser action was achieved in the neodymium ${ }^{4} F_{3 / 2} \rightarrow{ }^{4} I_{11 / 2}$ transition by butting plane mirrors to the end faces of the device and pumping a selected channel waveguide at $810 \mathrm{~nm}$. The lowest pump power required to reach threshold $(6 \mathrm{~mW})$ was found for the 10-mm-long sample, corresponding to $4 \mathrm{~mW}$ of absorbed power with two high-reflectivity mirrors. With a $25 \%$ output coupler an absorbed power threshold of $11 \mathrm{~mW}$ and a slope efficiency of $33 \%$ were observed [Fig. 2(a)]. Lasing was observed across a broad wavelength range 1048-1056 $\mathrm{nm}$.

Laser action was also achieved in the neodymium ${ }^{4} F_{3 / 2} \rightarrow{ }^{4} I_{13 / 2}$ transition at $1356 \mathrm{~nm}$ at a threshold of $370 \mathrm{~mW}$ of the absorbed power. With a value of
$6.8 \times 10^{-21} \mathrm{~cm}^{2}$ for the entire cross section ${ }^{10}$ the theoretical threshold value should be $11.2 \mathrm{~mW}$ of absorbed power. We attribute this difference to the excitedstate absorption of the ${ }^{4} F_{3 / 2} \rightarrow{ }^{4} G_{7 / 2}$ transition. ${ }^{11}$

Measurement of the spatial mode profile of the output from the waveguide was performed with a silicon camera and computer-based evaluation software. It was observed that the laser output was in the fundamental spatial mode, with a Gaussian mode profile in both guided directions. A guided output spot size $\left(1 / e^{2}\right.$ intensity radius) of $4.4 \mu \mathrm{m} \times 6.9 \mu \mathrm{m}$ elongated in the plane of the silica layer was calculated from the measured image size on the camera, which is presented in Fig. 2(b). The numerical aperture of the waveguide was measured with far-field imaging with a $\mathrm{He}-\mathrm{Ne}$ laser and was found to be approximately symmetrical with a value of 0.045 .

Propagation loss in the channel waveguide in the 1050-nm region was estimated from the slope efficiency and the lasing threshold results. From the threshold results and an estimated value of $1.9 \times 10^{-20} \mathrm{~cm}^{2}$ for the emission cross section ${ }^{1}$ a value of $0.08 \mathrm{~cm}^{-1}$ was calculated for the propagation loss coefficient $\alpha$ with two high-reflectivity mirrors and $0.06 \mathrm{~cm}^{-1}$ with a $25 \%$ output coupler, corresponding to a waveguide propagation loss of $<0.3 \mathrm{~dB} / \mathrm{cm}$. This result was confirmed with a separate calculation on the slope efficiency, yielding an upper limit for the propagation loss of $0.08 \mathrm{~dB} / \mathrm{cm}$. As a check on our evaluation of the propagation loss, we also measured

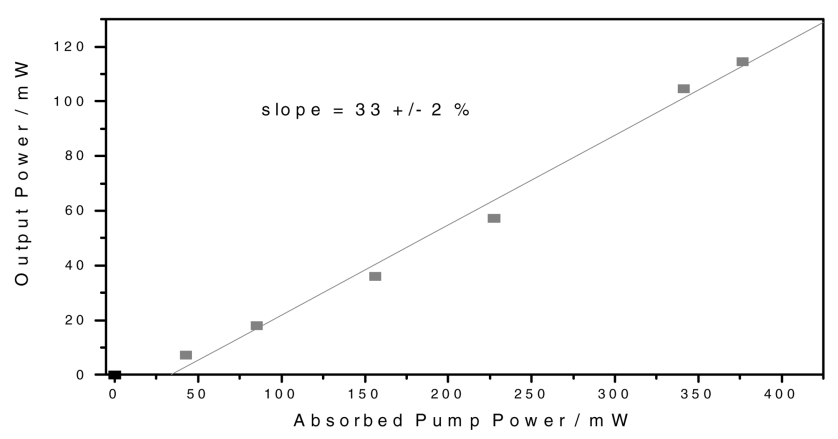

(a)

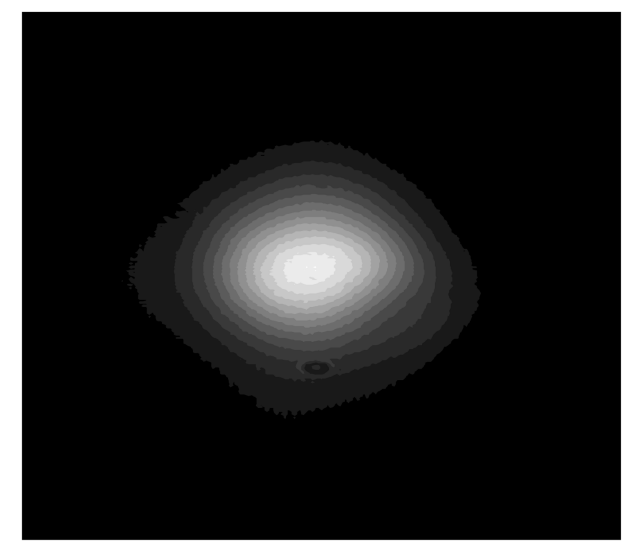

(b)

Fig. 2. (a) Output power versus absorbed pump power for a 10-mm-long sample with a $25 \%$ output coupler. (b) Spatial mode profile of the output from the waveguide. 
the performance of the 18-mm-long waveguide, finding that the threshold increased to $21 \mathrm{~mW}$ for the highreflectivity mirrors. With a $25 \%$ output coupler a threshold of $28 \mathrm{~mW}$ and a slope efficiency of $24 \%$ were observed. These results were again consistent with a propagation loss of $0.8 \mathrm{~dB} / \mathrm{cm}$.

In conclusion, we have reported a fabrication technique for the production of low-threshold single-mode laser channel waveguides in Nd-doped silica-onsilicon substrates, with a lasing wavelength of 1048$1056 \mathrm{~nm}$. Characterization of the laser performance in the channel waveguides demonstrates milliwattorder laser thresholds, single spatial mode operation, and propagation losses $<0.8 \mathrm{~dB} / \mathrm{cm}$. The channel waveguide was also observed to lase at $1356 \mathrm{~nm}$. To our knowledge, this result represents the first silica-on-silicon direct-UV-written Nd-doped laser and demonstrates a planar waveguide laser in the silica-on-silicon format, which has good lasing characteristics but is also suitably photosensitive to allow UV definition of waveguides.

D. Guilhot's e-mail address is dag@orc.soton.ac.uk.

\section{References}

1. E. K. Mwarania, J. Wang, J. Lane, and J. S. Wilkinson, J. Lightwave Technol. 11, 1550 (1993).

2. K. J. Malone, N. A. Sanford, and J. S. Hayden, Electron. Lett. 29, 691 (1993).

3. C. B. E. Gawith, A. Fu, T. Bhutta, P. Hua, D. P. Shepherd, E. R. Taylor, P. G. R. Smith, D. Milanese, and M. Ferraris, Appl. Phys. Lett. 81, 3522 (2002).

4. Y. Hibino, T. Kitagawa, M. Shimizu, F. Hanawa, and A. Sugita, IEEE Photon. Technol. Lett. 1, 349 (1989).

5. M. Kawachi, Opt. Quantum Electron. 22, 391 (1990).

6. M. Svalgaard and M. Kristensen, Electron. Lett. 33, 861 (1997).

7. B. Wu and P. L. Chu, IEEE Photon. Technol. Lett. 7, 655 (1995).

8. K. Hattori, T. Kitagawa, Y. Ohmori, and M. Kobayashi, IEEE Photon. Technol. Lett. 3, 882 (1991).

9. J. B. Ainslie, S. P. Craig, and S. T. Davey, J. Lightwave Technol. 6, 287 (1988).

10. J. W. Miniscalco, in Rare-Earth-Doped Fiber Lasers and Amplifiers, M. J. F. Digonnet, ed. (Marcel Dekker, New York, 2001), p. 53.

11. I. P. Alcock, A. I. Ferguson, D. C. Hanna, and A. C. Tropper, Opt. Lett. 11, 709 (1986). 